\title{
A Matched Pair Analysis of Multicenter Longterm Follow-up After Split-Liver Transplantation With Extended Right Grafts
}

\author{
Michael W. Ross, ${ }^{1}$ Matteo Cescon, ${ }^{2}$ Roberta Angelico, ${ }^{3}$ Enzo Andorno, ${ }^{4}$ Giorgio Rossi, ${ }^{5}$
} Antonio Pinna, ${ }^{2}$ Luciano De Carlis, ${ }^{6}$ Umberto Baccarani, ${ }^{7}$ Umberto Cillo, ${ }^{8}$ Michele Colledan, ${ }^{9}$ Vincenzo Mazzaferro, ${ }^{10}$ Giuseppe Tisone, ${ }^{11}$ Massimo Rossi, ${ }^{12}$ Fabio Tuzzolino, ${ }^{13}$ Duilio Pagano, ${ }^{14}$ Salvatore Gruttadauria, ${ }^{14}$ George Mazariegos, ${ }^{15}$ Bruno Gridelli, ${ }^{14}$ and Marco Spada ${ }^{3}$

\begin{abstract}
${ }^{1}$ Department of Medicine, Beth Israel Deaconess Medical Center, Harvard Medical School, Boston, MA; ${ }^{2}$ Department of Medical and Surgical Sciences, University of Bologna, Bologna, Italy; ${ }^{3}$ Division of Abdominal Transplantation and Hepatobiliopancreatic Surgery, Bambino Gesù, Children's Research Hospital, Istituto di Ricovero e Cura a Carattere Scientifico, Rome, Italy; ${ }^{4}$ Department of Transplant Surgery, San Martino Hospital, University of Genoa, Genoa, Italy; ${ }^{5}$ Division of Liver Transplantation, Ca' Granda Maggiore Hospital; and ${ }^{10}$ Division of Gastrointestinal Surgery and Liver Transplantation, Istituto Nazionale Tumori of Milano, Istituto di Ricovero e Cura a Carattere Scientifico Foundation, University of Milan, Milan, Italy; ${ }^{6}$ Department of Multi-Disciplinary Surgery, Division of General, HBP and Transplantation Surgery, Niguarda Transplant Center, Niguarda Hospital, Milano-Bicocca University, Milan, Italy; ${ }^{7}$ Division of Liver Transplant, Department of Medical and Biological Sciences, University Hospital of Udine, Udine, Italy; ${ }^{8}$ Division of Hepatobiliary Surgery and Liver Transplantation, University of Padua, Padua, Italy; ${ }^{9}$ Division of Liver and Small Bowel Transplantation, Azienda Ospedaliera Papa Giovanni XXIII, Bergamo, Italy; ${ }^{11}$ Division of Organ Transplantation, Tor Vergata University, Rome, Italy; ${ }^{12}$ Department of General Surgery and Organ Transplantation, Umberto I Hospital, Sapienza University, Rome, Italy; ${ }^{13}$ Information Technology Department, Mediterranean Institute for Transplantation and Advanced Specialized Therapies, Palermo, Italy; ${ }^{14}$ Department for the Study of Abdominal Diseases and Abdominal Transplantation, Istituto di Ricovero e Cura a Carattere Scientifico-Mediterranean Institute for Transplantation and Advanced Specialized Therapies, Palermo, Italy; and ${ }^{15}$ Division of Pediatric Transplantation, Children's Hospital of Pittsburgh, Pittsburgh, PA
\end{abstract}

\begin{abstract}
Split-liver transplantation has been proposed as an alternative to whole liver (WL) transplantation to expand the donor pool, but studies comparing adult longterm outcomes between the 2 methods are conflicting and limited. This is the first Italian multicenter study that retrospectively analyzed 119 matched-pair recipients of whole and extended right grafts (ERGs) for longterm survival outcomes. In the overall population, WL recipients showed higher patient survival at 1 (93\% versus $73 \%), 5(87 \%$ versus $65 \%)$, and 10 years $(83 \%$ versus $60 \%)$ after transplantation compared with split-liver recipients $(P<0.001)$; graft survivals of WL recipients were also superior at 1 (90\% versus 76\%), 5 (84\% versus 57\%), and 10 years (81\% versus 52\%) posttransplant $(P<0.001)$. However, among the 81 matched pairs that survived the first posttransplant year, 5- and 10-year patient survivals were $90 \%$ and $81 \%$ for split recipients and $99 \%$ and $96 \%$ for whole recipients, respectively $(P=0.34)$. The 5 - and 10 -year graft survivals were also comparable: $87 \%$ and $77 \%$ for split recipients, and $86 \%$ and $82 \%$ for whole recipients $(P=0.86)$. Cox regression analysis identified donor age $>50$, donor-to-recipient weight ratio $<1$, retransplantation status, and United Network for Organ Sharing I-IIA status as risk factors for partial graft use. There were no significant differences in 5-year outcomes based on center volume. In conclusion, we demonstrate that adult liver transplantation with ERGs can achieve longterm success comparable with that of whole grafts in appropriate patients but should be selectively used in patients with risk factors.
\end{abstract}

Liver Transplantation 23 1384-1395 2017 AASLD.

Received February 24, 2017; accepted June 18, 2017.

There is an increasingly large gap between the number of patients on the liver transplantation waiting list and

Abbreviations: BMI, body mass index; $C I$, confidence interval; $D R W R$, donor-to-recipient weight ratio; ERG, extended right graft; $H A T$, hepatic artery thrombosis; HCC, hepatocellular carcinoma; $H C V$, hepatitis $C$ virus; HH, high-volume whole and split-liver programs; HL, high whole and low split-liver programs; HM, high the number of available organs. This shortage has spurred the development of technical variant methods such as split-liver transplantation (SLT) - in which a deceased donor liver is divided between 2 recipients, conventionally an extended right graft (ERG) given to an adult and a smaller left lateral segment (LLS) given to a child-in an effort to increase the number of available grafts. Despite its potential, the wider application of SLT is limited to a number of factors such as 
suitable donor for splitting procedure and logistical issues; the proportion of liver transplants using split or other reduced-sized grafts has been reported at under $6 \%$ in both Europe and the United States in recent years. ${ }^{(1-4)}$

SLT has been promoted primarily to benefit the pediatric liver transplant candidates, as a solution to the lack of suitable graft options for small recipients. The introduction of SLT has decreased the pediatric wait-list mortality ${ }^{(5-7)}$ and achieved great levels of success in pediatric patients. ${ }^{(8-11)}$ On the other hand, the ERG is a partial graft transplanted to adult recipients instead of a whole liver transplantation (WLT). Whether SLT has comparable outcomes with WLT in adults is still under debate. Even though split grafts are from "ideal" deceased donors, ERG has been considered a higher-risk graft due to potential prolonged cold ischemia times and other technical limitations. Multicenter registry data implicate SLT as a risk factor for worse outcomes, ${ }^{(5,9,12)}$ but a number of studies have found comparable rates of 1-, 3-, and 5 -year patient and graft survival between the 2 methods. ${ }^{(1,3,8,9,13-18)}$ Much less information is available on SLT outcomes beyond this time frame, though single centers using carefully selected donors and recipients have achieved 10-year patient outcomes similar to WLT. $(3,19)$ Differences in the occurrence of postoperative complications are also poorly understood because complications

whole and medium split-liver programs; HR, hazard ratio; ICU, intensive care unit; ISMETT, Mediterranean Institute for Transplantation and Advanced Specialized Therapies; LL, low whole and split-liver programs; LLS, left lateral segment; MELD, Model for End-Stage Liver Disease; MM, medium whole and split-liver programs; PNF, primary nonfunction; PT, prothrombin time; SLT, split-liver transplantation; UNOS, United Network for Organ Sharing; WL, whole liver; WLT, whole liver transplantation.

Address reprint requests to Marco Spada, M.D., Ph.D., F.E.B.S., Division of Abdominal Transplantation and Hepatobiliopancreatic Surgery, Bambino Gesù, Children's Research Hospital IRCCS, Piazza Sant'Onofrio 4, 00146 Rome, Italy. Telephone: 003906 68591; Fax: 003906 68591; Email: marco.spada@opbg.net

This study was partially funded by the University of Pittsburgh Dean's Summer Research Program.

Additional supporting information may be found in the online version of this article.

Copyright $($ (C) 2017 by the American Association for the Study of Liver Diseases.

View this article online at wileyonlinelibrary.com.

DOI 10.1002/lt.24808

Potential conflict of interests: Nothing to report. with SLT have been reported as higher in some studies ${ }^{(3,20)}$ but comparable in others. ${ }^{(1,9,13,15,16,18)}$

A multicenter, longterm comparison of the outcomes of SLT and WLT in Italian recipients has never been reported. Our study is a retrospective matched-pair analysis comparing adult recipients of whole livers (WLs) to those of ERGs. This report, dating back to the first SLT procedures in Italy, presents the longterm outcomes as well as an analysis of donor and recipient clinical variables, based on data from 12 centers throughout the country.

\section{Patients and Methods}

\section{STUDY POPULATION}

This study includes data from 12 of the overall 18 Italian centers that performed liver transplants between 1997 and 2001-including San Martino Hospital of Genoa; Maggiore Hospital, Niguarda Hospital, and Istituto Nazionale Tumori Hospital of Milan; the University Hospitals of Bologna; University of Udine; University of Padua, Papa Giovanni XXIII Hospital of Bergamo; Tor Vergata and Sapienza University Hospitals of Rome; the Mediterranean Institute for Transplantation and Advanced Specialized Therapies (ISMETT) of Palermo. These centers performed over 90\% of the splitting procedures and WLTs in Italy during that time period. Pediatric liver transplantations were performed in 3 out of 12 participating centers: Milan Maggiore, Padua, and Bergamo.

The donor organs were allocated according to Italian National Transplant Center policy. With the exception of United Network for Organ Sharing (UNOS) I status patients, who were part of a national allocation process, donor livers were allocated on a regional basis. Within each region, organs were assigned in rotation to the constituent transplant centers, which then chose a recipient from their waiting list according to UNOS status. The assigned center, informed of potential pediatric recipients, determined if a liver should be split. Then, the center decided which partial graft they wanted to transplant: ERG, LLS, or both; if only 1 of the 2 grafts was used by the center, the remaining graft was reallocated following the criteria above. If a graft could not be allocated within the region, it was offered and allocated to a center in a different region. The splitting criteria adopted in Italy during the study period have been previously described by the authors ${ }^{(21)}$; those included recipient (donor-recipient body weight ratio, UNOS status) and donor characteristics (liver function tests, hemodynamic, and metabolic 
status), and liver macroscopic appearance. Age alone was not an exclusion criterion.

During the study period, 125 conventional split-liver procedures were performed in 101 adult and 24 pediatric cadaveric donors. The liver was divided into a LLS (segments II and III) and an ERG (segments I plus IV-VIII) as described in a previous report. ${ }^{(21)}$ The 121 splitting procedures were performed in situ; in a heartbeating, deceased donor; and the remaining 4 were performed ex situ, at the recipient center. Also, 124 patients (3 adult, 121 pediatric) received an LLS graft, and 125 (121 adult, 4 pediatric) received an ERG. One LLS graft was not transplanted due to iatrogenic lesions.

Using data from the Italian National Transplant Center, all adult WLT recipients transplanted between 1997 and 2001 in the enrolled centers were included in a pool of potential controls. From this pool, a control WLT recipient was matched 1:1 with each of the 121 adult ERG recipients based on the following characteristics: center SLT volume, recipient age, urgency of transplantation, indication for transplantation, number of past transplantations, and donor characteristics. An appropriate WLT matched-control recipient was not found for 2 ERG recipients; these patients were excluded from the study. Hence, our study focuses on 119 matched pairs, each with 1 ERG and 1 WLT adult recipient.

As this study aims to analyze the longterm outcomes, we conducted separate analyses for the overall population $(n=119)$ and for the matched pairs recipients surviving the first year after transplant, who were defined as the longterm study group ( $n=81$; Fig. 1$)$. The study protocol received a priori approval by the appropriate institutional review committee.

\section{CLINICAL VARIABLES OF INTEREST}

From the donor records, sex, age, ABO blood group, weight, length of intensive care unit (ICU) stay, cause of death, and administration of amines were recorded.

Recipients' variables including sex, age, ABO blood group, weight, body mass index (BMI), donor-torecipient weight ratio (DRWR), UNOS status, waitlist time, retransplantation status, indication for liver transplantation, degree of ascites and portosystemic encephalopathy, prothrombin time (PT), serum albu$\mathrm{min}$, and serum total bilirubin levels were collected.

The following postoperative variables were recorded: cold ischemia time, ICU stay time, total hospital stay, immunosuppressive medication, patient and graft survival, cause of death, cause of retransplantation, follow- up time, need for posttransplant dialysis, and presence of primary graft nonfunction.

In order to evaluate the possible impact of centers' volume of activity, liver transplantation programs were stratified according to the number of WLTs (high volume, $>50$ procedures; medium volume, 25-50 procedures; low volume, $<25$ procedures), and SLT (high volume, >20; medium volume, 10-20; low volume, $<10)$ per year during the study period.

\section{STATISTICAL ANALYSIS}

Descriptive statistics were performed using Microsoft Excel, testing for differences between the 2 patient groups with a Student $t$ test and chi-square test for continuous and categorical variables, respectively. For all groups studied, patient and graft Kaplan-Meier survival curves were generated with SPSS software, and the 2 groups were compared with the log-rank test. Patient and graft survivals were analyzed at 1,5 , and 10 years of follow-up for the overall population and at 5 and 10 years for the longterm study group. To find risk factors associated with undergoing SLT in place of WLT, each of the patient variables recorded were analyzed with univariate Cox regression to estimate a hazard ratio (HR) for patient loss and graft loss with 95\% confidence intervals (CIs). For the longterm group, patients who survived but required retransplantations within the first postoperative year were included in the Cox regression analysis of variables related to longterm patient loss but excluded from the longterm graft loss analysis sample. Multivariate Cox regression analysis was then performed on significant risk factors identified from univariate analysis to determine independent predictors of patient and graft loss after adjusting for covariables. A $P$ value of $<0.05$ was considered to be statistically significant.

\section{ETHICS APPROVAL}

This study was approved by the ISMETT and University of Pittsburgh institutional review boards (IRRB/03/13).

\section{Results}

\section{PREOPERATIVE AND POSTOPERATIVE VARIABLES}

A summary of the clinical variables for ERG and WLT recipients in both the overall population and the longterm study group is presented in Table 1 . 


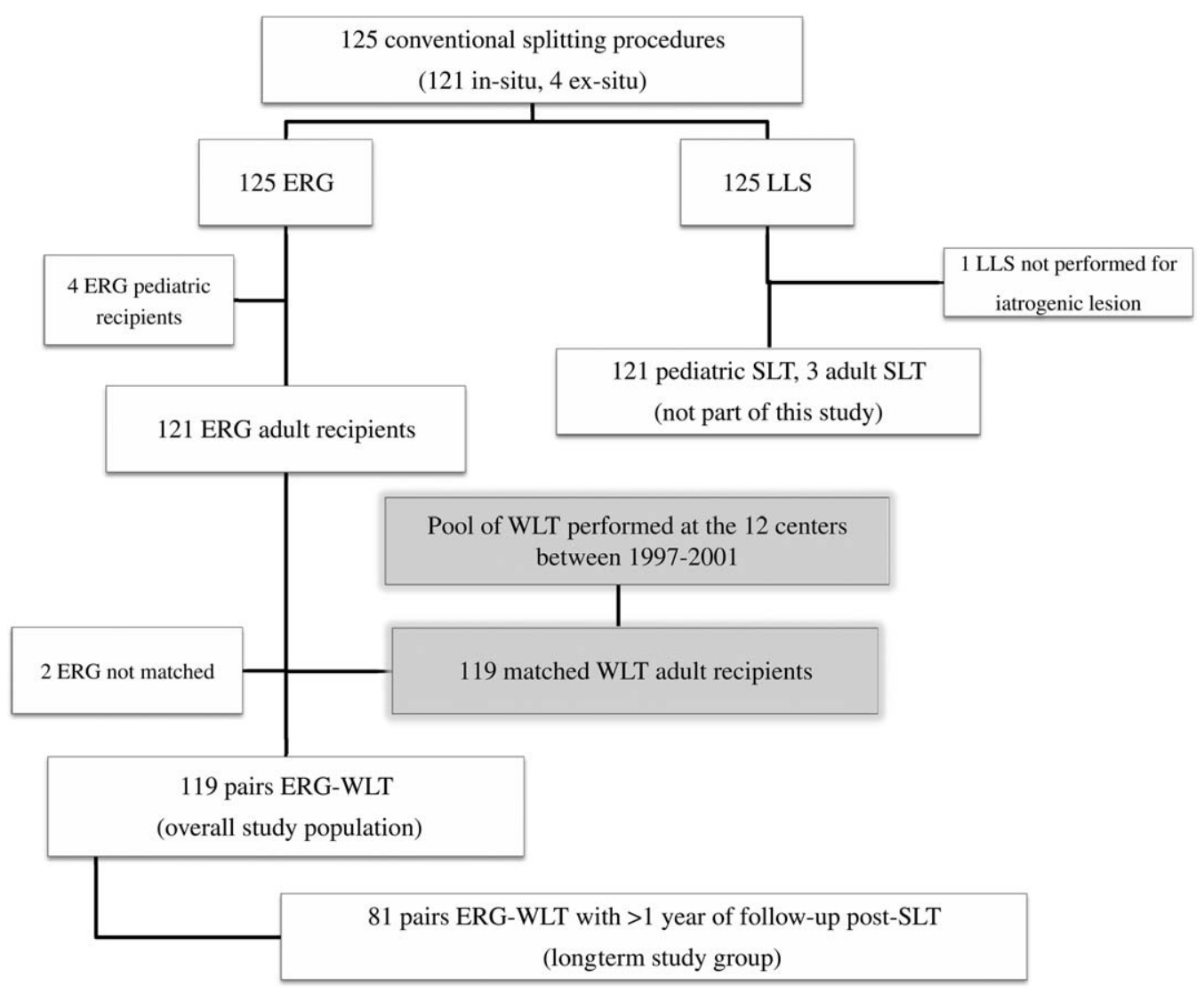

FIG. 1. Selection process of the study population.

In the overall population, administration of amines was higher for WL donors $(P<0.001)$. Recipient BMI was lower for split-liver recipients $(P=0.003)$, but there was no significant difference in DRWR $(P=0.57)$. SLT donor/recipient pairs were more likely to have identical ABO blood groups $(P<0.001)$. No other variations between the 2 populations were statistically significant.

In the longterm survival group, similar to the overall population study group, the SLT group displayed less donor administration of amines $(P<0.001)$, higher BMI $(P=0.01)$, with no difference in DRWR $(P=0.49)$, and more donor/recipient pairs with identical ABO blood groups $(P=0.006)$.

On the basis of transplant volume, the distribution of the participating transplant programs is described in Table 2. Preoperative and postoperative variables by center volume for the overall study population and for the longterm survival group can be found in Supporting Tables 1 and 2.

\section{PATIENT AND GRAFT SURVIVAL}

The median follow-up time for the entire population was 88 months (range 1-167 months). Kaplan-Meier survival curves for the entire population and the longterm group are shown in Figs. 2 and 3, respectively.

In the overall population, WLT recipients showed higher patient survival rates at 1 (93\% versus 73\%), 5 (87\% versus 65\%), and 10 years (83\% versus 60\%) compared with split-liver recipients $(P<0.001)$. WLT recipients had also superior graft survival rates compared with ERG recipients at 1 (90\% versus 76\%), 5 (84\% versus $57 \%)$, and 10 years (81\% versus 52\%; $P<0.001)$.

In the longterm cohort, the patient survival rates at 5 (99\% versus 90\%) and 10 years (86\% versus $81 \%)$ were similar for patients receiving WLT and ERG $(P=0.34)$. Also the graft survival rates in the WLT and ERG at $5(86 \%$ versus $87 \%)$ and 10 years $(82 \%$ versus $77 \%)$ were comparable $(P=0.86)$. 
TABLE 1. Donor and Recipient Clinical Characteristics in the Overall Population and in the Longterm Study Groups

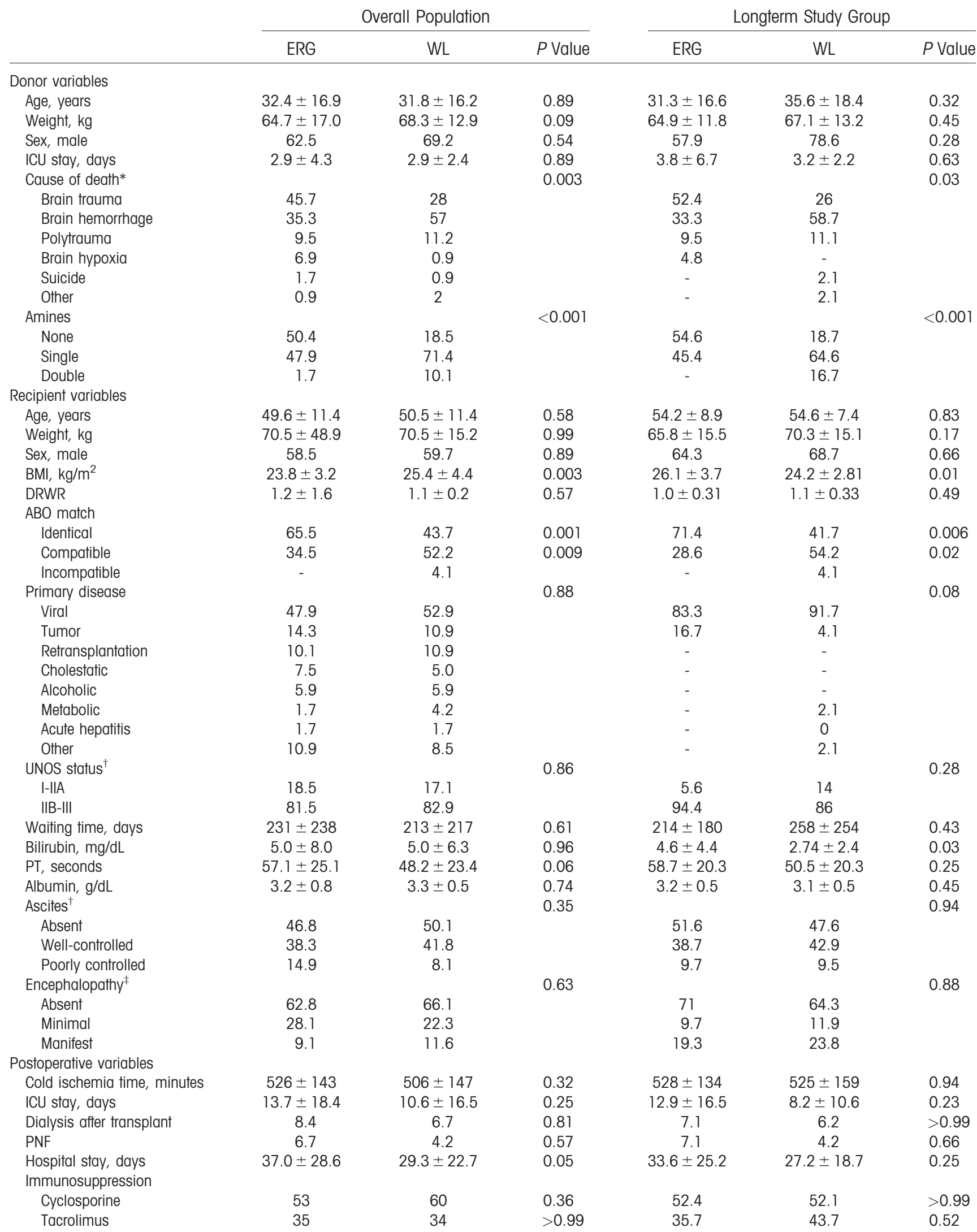

NOTE: Data are given as mean \pm standard deviation and percentage.

*Data available in 106 split-liver donors for overall study population. Data available in 62 split-liver recipients and in 76 WL recipients for longterm study group.

${ }^{\dagger}$ Data available in 103 split-liver recipients and in $105 \mathrm{WL}$ recipients for overall study population. Data available in 73 split-liver recipients and in $70 \mathrm{WL}$ recipients for longterm study group.

${ }^{\ddagger}$ Data available in 87 split-liver recipients and in 99 WL recipients for overall study population. Data available in 62 split-liver recipients and in $67 \mathrm{WL}$ recipients for longterm study group. 
TABLE 2. Distributions of the Participating Transplant Programs According to Their Volume Activity

\begin{tabular}{lcccc}
$\begin{array}{c}\text { Volume of } \\
\text { activity }\end{array}$ & $\begin{array}{c}\text { Number of } \\
\text { centers }\end{array}$ & $\begin{array}{c}\text { Number of } \\
\text { grafts }\end{array}$ & ERG & WLT \\
\hline HH & 2 & $85(36 \%)$ & 61 & 24 \\
HM & 2 & $24(10 \%)$ & 12 & 12 \\
HL & 2 & $52(22 \%)$ & 10 & 42 \\
MM & 2 & $56(24 \%)$ & 27 & 29 \\
LL & 4 & $21(8 \%)$ & 9 & 12
\end{tabular}

By log-rank test, there were no significant differences in 5-year patient or graft survivals among participating centers according to their volume of activity (Table 3).

\section{PATIENT AND GRAFT LOSS}

The causes of death and retransplantation are summarized in Table 4. Out of 49 ERG recipients' deaths, 17 (34.7\%) patients died more than 1 year after transplantation (51.7 \pm 34.4 months), whereas 15 (65.2\%) WLT recipients died in the long term, after an average of $76.1 \pm 38.7$ months. In both groups, recurrence of primary disease-hepatocellular carcinoma (HCC) or hepatitis $\mathrm{C}$ virus $(\mathrm{HCV})$-was the most common
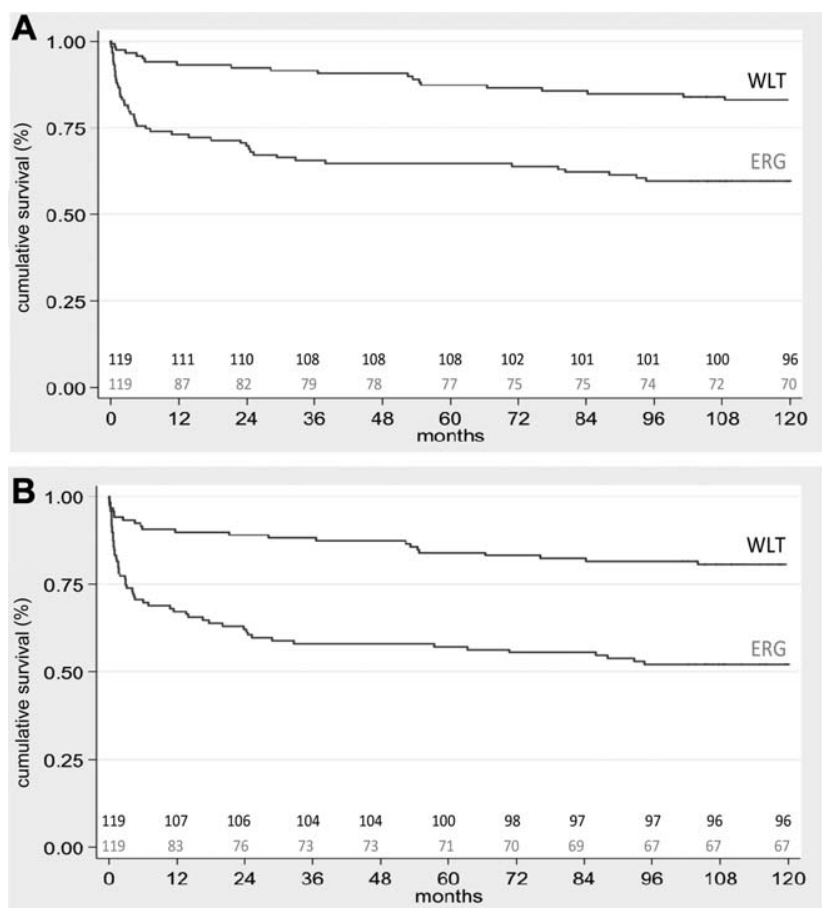

FIG. 2. (A) Patient and (B) graft survival curves of the overall study population.
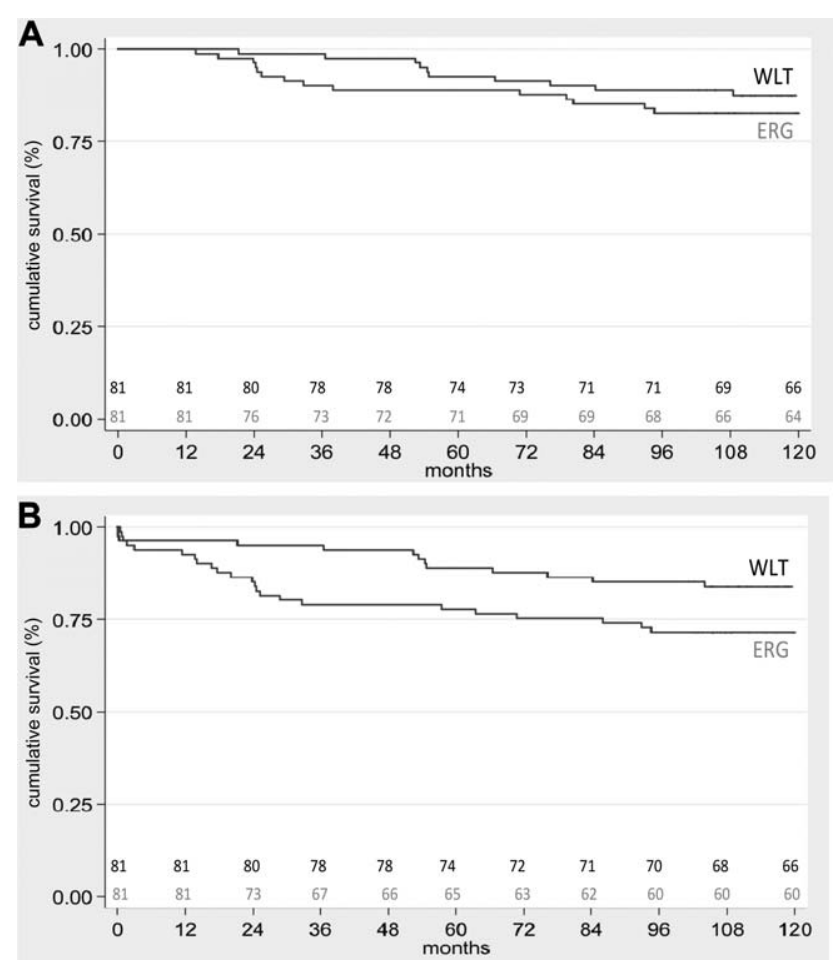

FIG. 3. (A) Patient and (B) graft survival curves of the longterm study groups.

cause of longterm death (35\% for ERG versus 27\% for WLT recipients).

Among ERG recipients, the overall retransplantation rate was $23.5 \%$, compared with $6.7 \%$ in the WLT cohort $(P<0.001)$. In the longterm population, 9 (11.1\%) ERG recipients were retransplanted mainly due to biliary complications (44.4\%), whereas 1 (1.2\%) WLT recipient was retransplanted after 104 months.

\section{COX REGRESSION ANALYSIS}

Among the overall study population, univariate analysis identified the following significant risk factors for graft loss: nonidentical versus identical $\mathrm{ABO}$ blood group $(\mathrm{HR}=2.74, P=0.01)$, donor age $>50$ versus $\leq 50$ years $(\mathrm{HR}=1.64, P=0.04)$, UNOS I-IIA status versus IIB-III status $(\mathrm{HR}=2.38, P=0.002)$, and retransplantation versus overall indication for transplantation $(\mathrm{HR}=2.13, P=0.01)$. At multivariate analysis, donor age $>50$ versus $\leq 50$ years $(\mathrm{HR}=1.03$, $P=0.005)$ was associated with higher risk of graft failure (Table 5).

At univariate analysis, patient survival was associated with the following risk factors: UNOS I-IIA status 
TABLE 3. Log-Rank Test Comparing 5-Year Patient and Graft Survivals Among Participating Centers, Stratified According to Their Volume of Activity

\begin{tabular}{lcc} 
& \multicolumn{2}{c}{ Log-Rank Value } \\
\cline { 2 - 3 } Study Group & $\begin{array}{c}\text { 5-Year Patient } \\
\text { Survival }\end{array}$ & $\begin{array}{c}5 \text {-Year Graft } \\
\text { Survival }\end{array}$ \\
\hline Overall study population $(n=238)$ & 0.0749 & 0.3197 \\
ERG recipients $(n=119)$ & 0.3144 & 0.3144 \\
WLT recipients $(n=119)$ & 0.2708 & 0.0992 \\
Overall longterm group $(n=162)$ & 0.1943 & 0.3822 \\
ERG recipients $(n=81)$ & 0.1494 & 0.1494 \\
WLT recipients $(n=81)$ & 0.7527 & 0.6651
\end{tabular}

versus IIB-III status $(\mathrm{HR}=2.19, P=0.01)$, donor age $>50$ versus $\leq 50$ years $(\mathrm{HR}=1.57, P=0.04)$, and retransplantation versus overall indication for transplantation ( $\mathrm{HR}=2.35, P=0.006)$. Donor age $>50$ versus $\leq 50$ years $(\mathrm{HR}=1.02, P=0.02)$ independently influenced patient survival according to multivariate regression analysis (Supporting Table 3).

For the longterm groups, DRWR over versus under $1(\mathrm{HR}=0.34, P=0.01)$ was found to be a risk factor according to univariate analysis. Multivariate analysis did not identify any independent risk factors for graft loss (Table 6).

At univariate analysis, patient loss was associated with donor age $>50$ versus $\leq 50$ years $(\mathrm{HR}=2.12$, $P=0.04)$ and DRWR over versus under 1 (HR $=0.33, P=0.04)$. Multivariate analysis identified only donor age $>50$ versus $\leq 50$ years $(\mathrm{HR}=1.05$, $P=0.04$ ) as a risk factor for patient loss (Supporting Table 4).

\section{Discussion}

The principal aims of our study were to compare longterm outcomes between split and WLT, as well as identify risk factors affecting longterm survival to improve donor and recipient selection. The retrospective and multicentric nature of our study made it difficult to adjust for differences in SLT recipient selection and management. Though there may be bias from specific center practices, the multicenter perspective offers the advantage of more universal applications. The case and control groups created by the matched-pair method had only minimal and expected differences in clinical variables, as addressed below.

The current study analyzes the very early experience with SLT, including the first procedures performed in Italy, drawing from multiple centers with varying levels of experience. At the time of the study, adult living donor liver transplantations (LDLTs) were not performed in Italy yet, so the technique had not impacted the learning curve of SLT. Recent case studies from Italian centers have demonstrated comparable outcomes between SLT and WLT, ${ }^{(17,18,22)}$ supporting the existence of a learning curve as established in other counties. ${ }^{(23,24)}$

An increase in SLT has the potential to provide greater access to donor organs-specifically the LLS graft-to pediatric recipients. Notably, wait-list mortality is higher for infants than any other age. ${ }^{(25,26)}$ SLT has been demonstrated to achieve similar outcomes to WLT and LDLT but, unlike LDLT, does not place a donor at risk. ${ }^{(9,11)}$

TABLE 4. Causes of Death and Retransplantation in the Overall Population and in the Longterm Study Groups

\begin{tabular}{|c|c|c|c|c|c|c|}
\hline & \multicolumn{3}{|c|}{ ERG } & \multicolumn{3}{|c|}{ WLT } \\
\hline & Total & Within 1 Year & After 1 Year & Total & Within 1 Year & After 1 Year \\
\hline Causes of death & $49(41.2)$ & $32(26.9)$ & $17(14.3)$ & 23 (19.3) & $8(6.7)$ & $15(12.6)$ \\
\hline PNF & $4(12.5)$ & $4(12.5)$ & - & - & - & - \\
\hline Vascular complication & $5(10.2)$ & $3(9.4)$ & $2(11.8)$ & - & - & - \\
\hline Infection/sepsis & $11(22.4)$ & $11(34.4)$ & - & $3(13)$ & $1(12.5)$ & $2(13.3)$ \\
\hline Multiple organ failure & $8(16.3)$ & $8(25)$ & - & $3(13)$ & $3(37.5)$ & - \\
\hline HCC recurrence & $3(6.1)$ & $1(3.1)$ & $2(11.8)$ & - & - & - \\
\hline HCV recurrence & $4(8.2)$ & - & $4(23.5)$ & $4(17.4)$ & - & $4(26.7)$ \\
\hline Biliary complication & - & - & - & $1(4.3)$ & - & $1(6.7)$ \\
\hline Other & $11(22.4)$ & $5(15.6)$ & $6(35.3)$ & $10(43.5)$ & $4(50)$ & $6(40)$ \\
\hline Unknown & $3(6.1)$ & - & $3(17.6)$ & $2(8.7)$ & - & $2(13.3)$ \\
\hline Causes of retransplantation & $28(23.5)$ & $19(16)$ & $9(7.6)$ & $8(6.7)$ & $7(5.9)$ & $1(0.8)$ \\
\hline PNF & $3(10.7)$ & $3(15.8)$ & - & $2(25)$ & $2(28.6)$ & - \\
\hline HAT & $12(42.9)$ & $11(57.9)$ & $1(11.1)$ & $2(25)$ & $2(28.6)$ & - \\
\hline Biliary complications & $6(21.4)$ & $2(10.5)$ & $4(44.4)$ & - & - & - \\
\hline Primary sclerosing cholangitis recurrence & $1(3.6)$ & - & $1(1.1)$ & & & \\
\hline Others & $4(14.3)$ & $1(5.3)$ & $3(33.3)$ & $3(37.5)$ & $3(42.9)$ & - \\
\hline Unknown & $2(7.1)$ & $2(10.5)$ & - & $1(12.5)$ & - & $1(100)$ \\
\hline
\end{tabular}

NOTE: Data are given as $\mathrm{n}(\%)$. 
TABLE 5. Univariate and Multivariate Analysis of Risk Factors Affecting Graft Loss in the Entire Study Population

\begin{tabular}{|c|c|c|c|c|c|}
\hline Risk Factor & HR & Standard Error & z & $P$ Value & $95 \% \mathrm{Cl}$ \\
\hline \multicolumn{6}{|l|}{ Univariate analysis } \\
\hline Donor age $>50$ versus $\leq 50$ years & 1.64 & 0.39 & 2.09 & 0.04 & $1.03-2.60$ \\
\hline Donor amine $0 \mathrm{mg} / \mathrm{ml} / \mathrm{kg}$ versus $1.2 \mathrm{mg} / \mathrm{ml} / \mathrm{kg}$ & 0.75 & 0.26 & -0.84 & 0.40 & $0.39-1.47$ \\
\hline ABO identical versus unidentical & 2.74 & 1.1 & 2.53 & 0.01 & $1.25-6.01$ \\
\hline DRWR over versus under 1 & 0.7 & 0.18 & -1.4 & 0.16 & $0.43-1.15$ \\
\hline \multicolumn{6}{|l|}{ Cold ischemia time } \\
\hline$<6$ versus $\geq 6$ hours & 0.94 & 0.38 & -0.17 & 0.87 & $0.43-2.05$ \\
\hline$<7$ versus $\geq 7$ hours & 1.08 & 0.3 & 0.27 & 0.79 & $0.62-1.87$ \\
\hline$<8$ versus $\geq 8$ hours & 0.73 & 0.19 & -1.2 & 0.23 & $0.44-1.22$ \\
\hline Recipient age $>50$ versus $\leq 50$ years & 0.87 & 0.21 & -0.58 & 0.57 & $0.55-1.38$ \\
\hline \multicolumn{6}{|l|}{ Primary disease } \\
\hline Cholestatic versus overall & 0.93 & 0.48 & -0.14 & 0.89 & $0.34-2.56$ \\
\hline Alcoholic versus overall & 0.66 & 0.39 & -0.7 & 0.48 & $0.21-2.10$ \\
\hline Viral versus overall & 0.76 & 0.18 & -1.16 & 0.25 & $0.47-1.21$ \\
\hline Metabolic versus overall & 0.37 & 0.38 & -0.98 & 0.33 & $0.05-2.68$ \\
\hline Tumor versus overall & 0.41 & 0.42 & -0.88 & 0.38 & $0.06-2.97$ \\
\hline Retransplantation versus overall & 2.13 & 0.64 & 2.52 & 0.01 & $1.18-3.84$ \\
\hline Other versus overall & 0.87 & 0.45 & -0.26 & 0.79 & $0.32-2.40$ \\
\hline UNOS I-IIA versus IIB-III & 2.38 & 0.67 & 3.1 & 0.002 & $1.38-4.12$ \\
\hline Cyclosporine versus tacrolimus & 0.9 & 0.23 & -0.42 & 0.67 & $0.54-1.49$ \\
\hline \multicolumn{6}{|l|}{ Multivariate analysis } \\
\hline Donor age $>50$ versus $\leq 50$ years & 1.03 & 0.01 & 2.81 & 0.005 & $1.01-1.05$ \\
\hline ABO identical versus unindentical & 1.38 & 0.87 & 0.52 & 0.61 & $0.40-4.75$ \\
\hline DRWR over versus under 1 & 0.92 & 0.31 & -0.25 & 0.79 & $0.48-1.77$ \\
\hline UNOS IIB-III versus I-IIA & 0.85 & 0.43 & -0.33 & 0.75 & $0.32-2.28$ \\
\hline \multicolumn{6}{|l|}{ Primary disease } \\
\hline Retransplantation versus overall & 2.21 & 1.24 & 1.41 & 0.16 & $0.73-6.63$ \\
\hline
\end{tabular}

Donor livers designated for splitting, as well as potential recipients of split grafts, must meet criteria concerning age, BMI, liver function, prior hospitalizations, and other factors. ${ }^{(2,27,28)}$ Accordingly, SLT donors are more likely to be hemodynamically stable, thus not requiring administration of amines, and more likely to have identical blood types to their recipients. Also, ERG recipients would be expected to have a lower BMI in order to attain a sufficiently high DRWR; there should be no significant difference in DRWR between the 2 groups, as was the case in our study.

TABLE 6. Univariate and Multivariate Analysis of Risk Factors Affecting Graft Loss in the Longterm Study Groups

\begin{tabular}{|c|c|c|c|c|c|}
\hline Risk Factor & HR & Standard Error & z & $P$ value & $95 \% \mathrm{Cl}$ \\
\hline \multicolumn{6}{|l|}{ Univariate analysis } \\
\hline Donor age $>50$ versus $\leq 50$ years & 2.08 & 0.78 & 1.95 & 0.052 & $1.00-4.33$ \\
\hline Donor amine 0 versus 1.2 & 0.86 & 0.46 & -0.28 & 0.78 & $0.30-2.47$ \\
\hline ABO identical versus unidentical & 2.96 & 1.82 & 1.77 & 0.08 & $0.89-9.85$ \\
\hline DRWR over versus under 1 & 0.34 & 0.15 & -2.48 & 0.01 & $0.15-0.80$ \\
\hline \multicolumn{6}{|l|}{ Cold ischemia time } \\
\hline$<6$ hours versus $\geq 6$ hours & 0.75 & 0.55 & -0.39 & 0.69 & $0.18-3.17$ \\
\hline$<7$ hours versus $\geq 7$ hours & 1.17 & 0.52 & 0.35 & 0.73 & $0.49-2.78$ \\
\hline$<8$ hours versus $\geq 8$ hours & 0.86 & 0.35 & -0.39 & 0.69 & $0.39-1.89$ \\
\hline Recipient age $>50$ versus $\leq 50$ years & 0.99 & 0.37 & -0.02 & 0.99 & $0.48-2.06$ \\
\hline \multicolumn{6}{|l|}{ Primary disease } \\
\hline Cholestatic versus overall & 0.55 & 0.56 & -0.58 & 0.56 & $0.07-4.09$ \\
\hline Alcoholic versus overall & 1.31 & 0.97 & 0.37 & 0.72 & $0.31-5.59$ \\
\hline Viral versus overall & 0.81 & 0.34 & -0.49 & 0.62 & $0.36-1.86$ \\
\hline Metabolic versus overall & 0.74 & 0.76 & -0.30 & 0.77 & $0.10-5.48$ \\
\hline Retransplantation versus overall & 3.31 & 3.40 & 1.17 & 0.24 & $0.44-24.7$ \\
\hline Other versus overall & 1.00 & 0.74 & 0.01 & 0.99 & $0.24-4.27$ \\
\hline UNOS I-IIA versus IIB-III & 1.87 & 1.02 & 1.15 & 0.25 & $0.64-5.42$ \\
\hline Cyclosporine versus tacrolimus & 0.82 & 0.32 & -0.52 & 0.60 & $0.38-1.76$ \\
\hline \multicolumn{6}{|l|}{ Multivariate analysis } \\
\hline Donor age $>50$ versus $<50$ years & 1.03 & 0.02 & 1.61 & 0.11 & $0.99-1.06$ \\
\hline DRWR over versus under 1 & 0.5 & 0.26 & -1.32 & 0.19 & $0.18-1.40$ \\
\hline
\end{tabular}


Our short-term results showed that recipients transplanted with ERG had inferior graft and patient survival rates compared with those who underwent WLT, mainly due to multiorgan failure and sepsis, suggesting these patients were likely not appropriate candidates for partial grafts. Improvements in short-term outcomes in more recent reports indicate much of this procedure-specific risk may now be obsolete given progression along the learning curve. ${ }^{(27)}$ Short-term outcomes among patients in North Italian centers, many of which are included in our cohort, have been extensively analyzed by Cardillo et al. ${ }^{(5)}$; accordingly, they are not the principal focus of this study and are not discussed further here.

This report is one of the few multicenter studies analyzing a large series and providing longterm results as far out as 10 years after transplantation. The 5 - and 10-year ERG patient and graft survival rates in our longterm cohort were comparable to that of WLT and consistent with survival rates published in other reports of WLT outcomes. ${ }^{(1,5,6,9,16,19,20)}$ Late deaths, unlike early deaths, were most frequently related to recurrence of primary disease, suggesting a distinct process warranting separate analysis. Previous multicenter studies have reported inferior outcomes to WLT in SLT patients. ${ }^{(5,9,12)}$ However, a recent meta-analysis showed similar patient and graft survival comparing right lobe SLT and WLT in adult patients, despite higher vascular and biliary complications in the splitliver recipients. ${ }^{(29)}$

Similar outcomes were achieved with a selected subgroup of patients, advocating identification of a suitable SLT recipient may be the strongest contributor to minimizing the risk of patient death and graft loss. Our results suggest that the use of ERG should be pursued in low-risk adult patients. Furthermore, more recent single-center studies demonstrate favorable outcomes with split grafts even with more liberal donor and recipient selection. ${ }^{(19,29)}$ Notably, transplantations in our study were performed before the introduction of the Model for End-Stage Liver Disease (MELD) score as a priority criterion for organ allocation. A recent study reported improved outcomes with split grafts after the introduction of the MELD score in the United States in 2002, suggesting a critical role of MELD in proper SLT recipient selection. ${ }^{(30)}$

Because center volume did not predict 5-year patient or graft survival, other explanations for variation between centers, such as SLT donor/recipient selection and posttransplant follow-up care plans, should be explored. The limited number of procedures at low-volume centers did not allow the comparison of individual center survivals. A total of 61 ERG procedures $(75 \%$ of the patients in our study with longterm follow-up) were performed by the 2 transplant centers defined as high-volume programs both for WLT and SLT, showing that, at the beginning of the Italian splitting experience, partial liver grafts were accepted/ performed mainly by high-volume transplant centers. Low-volume centers were likely more cautious regarding SLT recipient selection, which may have influenced their outcomes.

The results of our study and subsequent SLT experiences have been especially important in defining the new Italian national liver allocation criteria, which now redeem the shortcomings of an entirely MELD-based system, adopted since 2003, by considering donor characteristics and allowing for the potential assignment of partial grafts to less acutely ill patients. ${ }^{(31)}$ Nowadays, the national allocation policy considers all standard-risk donors below 50 years of age suitable for SLT. Unless an adult with UNOS status 1 or $M E L D \geq 30$ is on the waiting list, the LLS of a deceased donor with such characteristics is offered for pediatric liver transplantation. If the LLS is allocated to a pediatric recipient, the ERG is then allocated to the best-matching adult recipient based not only on the MELD score, but also on the recipient clinical status and the donor/recipient size-matching. ${ }^{(32)}$

Retransplantation status is another factor that can influence a patient's prognosis. In our study, the proportions of recipients in each group undergoing retransplantation were similar both to each other and to reported proportions of liver transplants worldwide. ${ }^{(33-36)}$ Our results suggest retransplantation represented a significant risk factor for patient and graft loss.

The majority of early retransplantations were due to hepatic artery thrombosis (HAT), which occurred more frequently in the SLT group due to the fact that the left hepatic artery and celiac trunk were left with the LLS, while only the right hepatic artery was included with the ERG, resulting in more difficult arterial anastomosis in ERG recipients. ${ }^{(21)}$ To minimize the risk of thrombosis, taking these data into account, the Italian split policy was recently revised so that to divide the hepatic artery properly according to donor and recipient anatomy rather than always keeping the celiac tripod with the LLS. ${ }^{(32)}$

Biliary complications were a cause of retransplantation in the ERG group but did not impact patient survival. Interestingly, biliary complications caused retransplantation in ERG recipients mainly after 1 year 
from transplantation. Diagnosing biliary complications may be complex in SLT and, as demonstrated in pediatric SLT recipients, early diagnosis and treatment with biopsy or cholangiography in the setting of impaired liver function, even without biliary tree dilation, avoids late graft failure secondary to biliary cirrhosis. ${ }^{(37)}$ In our study, diagnosis and management of biliary complications-for example, the use of $\mathrm{T}$ tubes-were different in each center and thus were not analyzed. Patients requiring retransplantation for biliary complications were clustered in 3 centers, suggesting this morbidity may be more a function of postoperative care than graft choice. Importantly, more recent series have demonstrated lower rates of vascular and biliary complications and retransplantation in SLT, with results comparable with those of WLT. ${ }^{(17,19)}$

Our study identified risk factors associated with patient and graft loss in ERG recipients, underscoring the importance of adequate donor/recipient matching for early graft and patient survival. With univariate analysis, risk factors for early partial graft failure were $\mathrm{ABO}$ mismatch, retransplantation, and $\mathrm{DRWR}<1$, and those for early patient loss were UNOS I-IIA status, retransplantation, and DRWR $<1$. In particular, we confirmed that UNOS I-IIA status, including patients with acute liver failure and patients admitted to the ICU with kidney failure or gastrointestinal bleeding, as well as ABO incompatibility were widely associated with worse transplantation outcomes, supporting prudent use of SLT in these patients as documented in previous reports. ${ }^{(5,6,9,27,38)}$

With multivariate analysis, the only significant risk factor impacting patient and graft survivals, both in the short and long term, was donor age of $>50$ years. Advanced donor age has previously been reported as a risk factor, though some studies demonstrate successes using livers from older donors. ${ }^{(5,27,28)}$ However, there is no established cutoff for advanced age, and longterm data on this subject are scarce..$^{(5,27,28)}$ In our series, 21 (17.6\%) ERG recipients underwent transplantation with grafts from donors over 50 years. The use of such donors was mainly related to the presence of very sick pediatric recipients on the waiting list. Splitting grafts from older donors did not influence the pediatric outcomes. As shown in a previous Italian report, ${ }^{(17)}$ adults transplanted with ERG had inferior graft survival rates compared with patients receiving whole grafts from donors over 50 years. This indicates that risk factors may be additive and that accurate assessment of their presence or absence is crucial for appropriate selection of candidate ERG recipients. Therefore, after those results, the new Italian splitting allocation policy considers only cadaveric donors with age $\leq 50$ years.

In our series, patients with $\mathrm{HCV}$ receiving ERG had similar outcomes to those transplanted with whole grafts (data not shown), suggesting these patients can have longterm success with partial grafts in the absence of other recognized risk factors. ${ }^{(39,40)}$ Thus, the transplantations in this study were performed before the newer direct-acting antiviral agents became available.

The lack of risk factor consistency across all study groups indicates the cause of graft loss is likely multifactorial and is consistent with previous work reporting that the effect of 1 risk factor can be minimized by controlling others. ${ }^{(27)}$ Therefore, the presence of only 1 risk factor above should not rule out the use of SLT.

In conclusion, SLT is still being explored as an alternative technique to expand the donor pool. Our multicenter study, which represents one of the earliest experiences with SLT, showed that ERG recipients were at an increased risk for early mortality and graft failure, though satisfactory longterm outcomes were achieved in those surviving the first posttransplant year. These results are mainly related to the SLT learning curve in Italy, where the use of partial grafts has progressively increased, especially in combined adult and pediatric transplant programs. Worse outcomes were associated with donor age $>50$ years, $\operatorname{DRWR}<1$, retransplantation, and recipient UNOS I-IIA status. ERG use should be more selective in the presence of these risk factors, especially in combination. Our results support the 5- and 10-year efficacy of ERG transplantation in appropriately selected adults and have helped define a novel national allocation program to safely increase sources of viable split grafts in the current era of organ shortage.

Acknowledgments: The authors would like to acknowledge Warren Blumberg for his assistance with language revision.

\section{REFERENCES}

1) Wilms C, Walter J, Kaptein M, Mueller L, Lenk C, Sterneck $\mathrm{M}$, et al. Long term outcome of split liver transplantation using right extended grafts in adulthood: a matched pair analysis. Ann Surg 2006;244:865-872.

2) Saidi RF, Jabbour N, Li Y, Shah SA, Bozorgzadeh A. Outcomes in partial liver transplantation: deceased donor split-liver vs. live donor liver transplantation. HBP (Oxford) 2011;13:797-801.

3) Vagefi PA, Parekh J, Ascher NL, Roberts JP, Freise CE. Outcomes with split liver transplantation in 106 recipients. Arch Surg 2011;146:1052-1059. 
4) Burroughs AK, Sabin CA, Rolles K, Delvart V, Karam V, Buckels J, et al.; European Liver Transplant Association. 3-month and 12-month mortality after first liver transplant in adults in Europe: predictive models for outcome. Lancet 2006;367:225-232.

5) Cardillo M, De Fazio N, Pedotti P, De Feo T, Fassati LR, Mazzaferro V, et al.; for NITp Liver Transplantation Working Group. Split and whole liver transplantation outcomes: a comparative cohort study. Liver Transpl 2006;12:402-410.

6) Hong JC, Yersiz H, Busuttil RW. Where are we today in split liver transplantation? Curr Opin Organ Transplant 2011;16: 269-273.

7) Merion RM, Rush SH, Dykstra DM, Goodrich N, Freeman RB Jr, Wolfe RA. Predicted lifetimes for adult and pediatric split liver versus adult whole liver transplant recipients. Am J Transplant 2004;4:1792-1797.

8) Nesher E, Island E, Tryphonopoulos P, Moon J, Nishida S, Selvaggi G, et al. Split liver transplantation. Transplant Proc 2011;43:1736-1741.

9) Hong JC, Yersiz H, Farmer DG, Duffy JP, Ghobrial RM, Nonthasoot B, et al. Long term outcomes for whole and segmental liver grafts in adult and pediatric liver transplant recipients: a 10-year comparative analysis of 2,988 cases. J Am Coll Surg 2009;208:682-689.

10) Yersiz H, Renz JF, Farmer DG, Hisatake GM, McDiarmid SV, Busuttil RW. One hundred in situ split liver transplantations: a single-center experience. Ann Surg 2003;238:496-505.

11) Gridelli B, Spada M, Petz W, Bertani A, Lucianetti A, Colledan $\mathrm{M}$, et al. Split liver transplantation eliminates the need for living-donor liver transplantation in children with end-stage cholestatic disease. Transplantation 2003;75:1197-1203.

12) Feng S, Goodrich NP, Bragg-Gresham JL, Dykstra DM, Punch JD, DebRoy MA, et al. Characteristics associated with liver graft failure: the concept of a donor risk index. Am J Transplant 2006; 6:783-790.

13) Broering DC, Topp S, Schaefer U, Fischer L, Gundlach M, Sterneck M, et al. Split liver transplantation and risk to the adult recipient: analysis using matched pairs. J Am Coll Surg 2012; 195:648-657.

14) Abecassis M, Superina R, Axelrod D. Split liver sharing in the United States. Am J Transplant 2005;5:1583-1584.

15) Sandroussi C, Crawford M, Lockwood DS, Tang P, Gallagher JP, Pleass $\mathrm{H}$, et al. Donor and recipient selection leads to good patient and graft outcomes for right lobe split transplantation versus whole graft liver transplantation in adult recipients. Liver Transpl 2009;15:1586-1593.

16) Takebe A, Schrem H, Ringe B, Lehner F, Strassburg C, Klempnauer J, Becker T. Extended right liver grafts obtained by an ex situ split can be used safely for primary and secondary transplantation with acceptable biliary mortality. Liver Transpl 2009;15:730-737.

17) Spada M, Cescon M, Aluffi A, Zambelli M, Guizzetti M, Lucianetti A, et al. Use of extended right grafts from in situ split livers in adult liver transplantation: a comparison with whole liver transplants. Transplant Proc 2005;37:1164-1166.

18) Baccarani U, Adani GL, Risaliti A, Sainz-Barriga M, Lorenzin D, Chiarandini P, et al. Long-term results of in situ split-liver transplantation. Transplant Proc 2005;37:2592-2594.

19) Doyle MB, Maynard E, Lin Y, Vachharajani N, Shenoy S, Anderson C, et al. Outcomes with split liver transplantation are equivalent to those with whole liver transplantation. J Am Coll Surg 2013;217:102-112.

20) Bonney GK, Aldouri A, Attia M, Lodge PA, Toogood GJ, Pollard SG, Prasad R. Outcomes in right liver lobe transplantation: a matched pair analysis. Transpl Int 2008;21:1045-1051.
21) Spada M, Gridelli B, Colledan M, Segalin A, Lucianetti A, Petz W, et al. Extensive use of split liver for pediatric liver transplantation: a single-center experience. Liver Transpl 2000;6:415-428.

22) Cintorino D, Spada M, Gruttadauria S, Riva S, Luca A, Volpes $\mathrm{R}$, et al. In situ split liver transplantation for adult and pediatric recipients: an answer to organ shortage. Transplant Proc 2006; 38:1096-1098.

23) Schrem H, Kleine M, Lankisch TO, Kaltenborn A, Kousoulas L, Zachau L, et al. Long-term results after adult ex situ split liver transplantation since its introduction in 1987. World J Surg 2014;38:1795-1806.

24) Battula NR, Platto M, Anbarasan R, Perera MT, Ong E, Roll $\mathrm{GR}$, et al. Intention to split policy: a successful strategy in a combined pediatric and adult liver transplant center. Ann Surg 2017;265:1009-1015.

25) Magee JC, Krishnan SM, Benfield MR, Hsu DT, Shneider BL. Pediatric transplantation in the United States: 1997-2006. Am J Transplant 2008;8(pt 2):935-945.

26) Kim WR, Smith JM, Skeans MA, Schladt DP, Schnitzler MA, Edwards EB, et al. OPTN/SRTR 2012 annual data report: liver. Am J Transplant 2014;14:69-96.

27) Lee KW, Cameron AM, Maley WR, Segev DL, Montgomery RA. Factors affecting graft survival after adult/child split liver transplantation: analysis of the UNOS/OPTN data base. Am J Transplant 2008;8:1186-1196.

28) Harring TR, O'Mahony CA, Goss JA. Extended donors in liver transplantation. Clin Liver Dis 2011;15:879-900.

29) Wan P, Li Q, Zhang J, Xia Q. Right lobe split liver transplantation versus whole liver transplantation in adult recipients: a systematic review and meta-analysis. Liver Transpl 2015;21: 928-943.

30) Cauley RP, Vakili K, Fullington N, Potanos K, Graham DA, Finkelstein JA, Kim HB. Deceased-donor split-liver transplantation in adult recipients: is the learning curve over? J Am Coll Surg 2013;217:672-684.

31) Cillo U, Burra P, Mazzaferro V, Belli L, Pinna AD, Spada M, et al.; for I-BELT (Italian Board of Experts in the Field of LiverTransplantation). A multistep, consensus-based approach to organ allocation in liver transplantation: toward a "blended principle model.” Am J Transplant 2015;15:2552-2561.

32) Linee Guida e Protocolli. http://www.crtsicilia.it/centri_trap/All_ 04_Protocollo_split_liver_00.pdf. Accessed July 28, 2017.

33) Yoo PS, Umman V, Rodriguez-Davalos MI, Emre SH. Retransplantation of the liver: review of current literature for decision making and technical considerations. Transplant Proc 2013;45: 854-859.

34) Pfitzmann R, Benscheidt B, Langrehr JM, Schumacher G, Neuhaus R, Neuhaus P. Trends and experiences in liver retransplantation over 15 years. Liver Transpl 2007;13:248-257.

35) Kashyap R, Jain A, Reyes J, Demetris AJ, Elmagd KA, Dodson $\mathrm{SF}$, et al. Causes of retransplantation after primary liver transplantation in 4000 consecutive patients: 2 to 19 years follow-up. Transplant Proc 2001;33:1486-1487.

36) Marudanayagam R, Shanmugam V, Sandhu B, Gunson BK, Mirza DF, Mayer D, et al. Liver retransplantation in adults: a single-centre, 25-year experience. HPB (Oxford) 2010;12:217-224.

37) Miraglia R, Maruzzelli L, Caruso S, Riva S, Spada M, Luca A, Gridelli B. Percutaneous management of biliary strictures after pediatric liver transplantation. Cardiovasc Intervent Radiol 2008; 31:993-998.

38) Kiuchi $T$, Kasahara $M$, Uryuhara $K$, Inomata $Y$, Uemoto $S$, Asonuma $\mathrm{K}$, et al. Impact of graft size mismatching on graft prognosis in liver transplantation from living donors. Transplantation 1999;67:321-327. 
39) Humar A, Horn K, Kalis A, Glessing B, Payne WD, Lake J. Living donor and split-liver transplants in hepatitis $\mathrm{C}$ recipients: does liver regeneration increase the risk for recurrence? Am J Transplant 2005;5:399-405.
40) Shiffman ML, Stravitz RT, Contos MJ, Mills AS, Sterling RK, Luketic VA, et al. Histologic recurrence of chronic hepatitis C virus in patients after living donor and deceased donor liver transplantation. Liver Transpl 2004;10:1248-1255. 